\title{
La Monnaie et la Banque, un transfert technologique et culturel au Japon au XIX ${ }^{\mathrm{e}}$ siècle
}

\section{Georges Depeyrot et Marina Kovalchuk}

\section{OpenEdition}

\section{Journals}

Édition électronique

URL : http://journals.openedition.org/rsl/208

DOI : $10.4000 /$ rsl.208

ISSN : 2271-6246

\section{Éditeur}

Éditions Rue d'Ulm

\section{Référence électronique}

Georges Depeyrot et Marina Kovalchuk, « La Monnaie et la Banque, un transfert technologique et culturel au Japon au XIX ${ }^{\mathrm{e}}$ siècle », Revue Sciences/Lettres [En ligne], 1 | 2013, mis en ligne le 15 janvier 2012, consulté le 30 avril 2019. URL : http://journals.openedition.org/rsl/208 ; DOI : 10.4000/rsl.208

Ce document a été généré automatiquement le 30 avril 2019

(c) Revue Sciences/Lettres 


\title{
La Monnaie et la Banque, un transfert technologique et culturel au Japon au XIX ${ }^{\mathrm{e}}$ siècle
}

\author{
Georges Depeyrot et Marina Kovalchuk
}

1 Le phénomène actuel de mondialisation de l'économie ressemble souvent, pour l'historien, à une répétition de situations anciennes. Le champ des économies monétaires est un lieu de transferts de compétence ou de technologie : il n'est pas de lien, politique ou économique, qui ne se concrétise par des échanges monétaires; il n'est pas de domination qui ne se traduise dans l'usage de la monnaie ; il n'est pas de partenariat qui n'inclue la monnaie quelle qu'en soit la forme.

2 Imposée ou recherchée, acceptée ou refusée, la monnaie (ou l'unité monétaire) est un des critères d'intégration ou de coopération économique entre deux ou plusieurs pays. De même que l'unification monétaire est un des critères de l'existence d'un État, l'appartenance de ce même État à une zone monétaire est un critère de son intégration au groupe des pays dominants.

3 Le cas du Japon du XIX siècle est d'autant plus intéressant en matière monétaire qu'il passe d'un régime féodal et local à une unification, puis à une intégration dans le groupe des pays à étalon-or. Dans le même temps, il transforme son économie, son armée, et, de pays colonisable, devient pays expansionniste puis colonisateur dès le début du $\mathrm{xx}^{\mathrm{e}}$ siècle (la Corée est annexée en 1910). Au-delà des faits, les dirigeants politiques japonais ont voulu expliciter leurs réformes pour crédibiliser l'entrée du Japon sur la scène internationale. Chaque réforme, chaque évolution a donc été détaillée, commentée et argumentée. Désireux de voir leur pays devenir une puissance économique et conscients de l'importance de la question monétaire, ils ont pallié leur manque de savoir-faire par l'importation des techniques occidentales. La question de la crédibilité de la monnaie et de la confiance en sa stabilité étant au cœur de toute relation financière, afin que le Japon puisse emprunter sur les plus grandes places bancaires (Paris ou Londres), ils ont relaté avec précision les améliorations qu'ils apportaient à ses systèmes monétaires et 
bancaires, calquées, au plus près, sur l'organisation des institutions respectables et respectées des pays dominants.

4 Afin de comprendre les modalités d'importation d'un système monétaire et bancaire et son implantation au Japon, nous étudions et confrontons des sources, souvent méconnues, qui ont l'intérêt de documenter des phénomènes de changement de stock monétaire dans le contexte d'une crise et, consécutivement, de l'unification monétaire puis du passage rapide du bimétallisme au monométallisme d'un pays développé.

Après l'arrivée en rade de Tokyo du commodore Perry, porteur d'un ultimatum demandant l'ouverture du pays aux étrangers (1853), après son retour à la tête d'une flotte militaire internationale (février 1854), le Japon avait été obligé de signer le 31 mars 1854 la Convention de Kanagawa ouvrant le pays, puis, dès 1858, des traités largement imposés par les puissances occidentales au shogunat des Tokugawa. Dans les années 1860, les Japonais prennent conscience du caractère irréversible de l'évolution. Dès 1862, une mission, dite Mission Takenouchi, est envoyée visiter les pays européens pour en rapporter des informations propres à nourrir la réflexion politique. Dès son arrivée au pouvoir ( 3 février 1867), l'empereur Mutsuhito s'entoure de ministres et de conseillers de haut niveau et entame une série de réformes destinées à moderniser le pays. En novembre, le shogun (seigneur local ou régional) d'Edo remet son pouvoir à l'empereur qui inaugure le 23 octobre 1868 l'ère Meiji, marquant la fin du régime féodal et le renouveau du pouvoir centralisé impérial. Il quitte alors Kyoto pour s'installer à Edo rebaptisée Tokyo. Plutôt que d'envisager un affrontement qui aurait certainement tourné au désavantage du Japon (comme ce fut le cas de la Chine), le Mikado préfère ouvrir le Japon aux Occidentaux et réformer le pays pour le doter d'une dimension internationale lui permettant de traiter d'égal à égal avec les grandes puissances.

En l'espace de quelques décennies, le régime transforme la vie politique (passant en substance d'un féodalisme à un régime parlementaire), créant une armée nationale (très rapidement capable de mener des opérations offensives), bouleversant la vie économique en changeant le système d'imposition, modernisant les communications. L'évolution fut lente (Parlement en 1889, constitution en 1890), mais irréversible. Pour mener toutes ces transformations, l'empereur s'appuie sur les exemples occidentaux. Il continue à envoyer plusieurs missions dont la plus célèbre est la mission Iwakura, qui parcourt les États-Unis et l'Europe pendant près de trois ans (1871-1873), rédigeant régulièrement des mémoires sur la vie politique et la technologie occidentales (ils ont été publiés en intégralité1). De très nombreux hommes politiques se rendent ainsi à l'étranger, soit pour effectuer leurs études, soit en mission. Ainsi, le marquis Ito Hirobumi séjourne pour ses études hors du Japon, il fait partie de la mission Iwakura avant d'assumer cinq fois la charge de Premier ministre. En 1870, Ito rédige à l'intention de l'empereur un long mémorandum intitulé "Raisons pour établir le nouveau système monétaire japonais selon le système

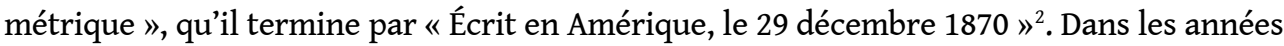
1860 , le comte Inouye Kaoru, avant de devenir ministre des affaires étrangères et viceministre des finances, et le marquis Ito Hirobumi, futur rédacteur de la constitution et premier Premier ministre de l'histoire du Japon, effectuent leurs études en Angleterre. L'ouverture et l'intégration économique du Japon est donc, avant tout, le résultat d'un transfert de compétence par la formation des élites du pays.

7 Tous ces hommes pensent que l'établissement d'un système monétaire moderne est alors indispensable à l'économie nippone, pouvant seul assurer une homogénéisation des impositions et permettre la préparation d'un budget d'État. Dès avril 1868, la décision est 
prise de frapper de nouvelles monnaies uniformes et de bonne qualité pour remplacer le stock hétérogène. Seul un transfert massif de technologie et de compétence peut permettre la création ex nihilo d'un stock monétaire.

8 Harry Parkes, ministre plénipotentiaire anglais, est saisi par l'empereur, le 30 mai 1868, d'une demande concernant la possibilité de créer un atelier monétaire. Des négociations s'ouvrent pour le rachat des presses (des James Watt de Londres) et du matériel de l'atelier de Hong Kong fermé au début de l'année 1868. Pour faire fonctionner l'atelier (qui nécessite une centaine d'hommes) capable de produire 35000 dollars d'argent de type mexicain en douze heures, le Japon embauche comme directeur Thomas Kinder, l'ancien directeur de l'atelier de Hong Kong. Ce choix fut un sujet de conflit, non en raison de la personnalité de Kinder, mais parce que les Japonais voulurent dans un premier temps assurer eux-mêmes la direction de l'atelier. À l'évidence, il convenait de s'adjoindre les services de l'ancienne équipe de la Monnaie de Hong Kong : constituer le stock monétaire pour 33 millions de Japonais n'était pas simple.

Enfin, le 14 octobre 1868, le matériel arrive au port d'Osaka, où l'atelier impérial, un bâtiment de style anglais, est en construction. Treize Européens accompagnent Kinder, qui prennent en charge les divers départements (essais, fonte, gravure des coins, pesée, frappe, acides, etc.). Le 4 avril 1871, l'atelier est inauguré et entre en fonction. Entre 1871 et le 31 janvier 1875, la frappe de la monnaie est entièrement entre les mains des Anglais, qui assurent des productions de qualité. Ainsi, en 1873-1874, l'atelier produit 2,319 millions de monnaies d'or, 24,532 millions de monnaies d'argent et 36 millions de monnaies de cuivre, toutes rigoureusement similaires.

Kinder et ses associés garantissent non seulement la bonne marche de l'atelier, mais aussi le transfert de compétence. Lorsque Kinder part avec sept autres directeurs le 31 janvier 1875, il laisse un atelier en ordre de marche «In every detail the Mint is now in the most perfect order, and will be left by me in that state " (rapport de Kinder, 28 janvier 18753). L'effectif de l'atelier augmente alors considérablement : 380 personnes en 1874, 556 en 1876 après son départ. La production augmente aussi, passant à 0,076 million de monnaies d'or, 23 millions d'argent et 87 millions de cuivre, soit 112 millions de monnaies en 1876 contre 62 millions en 1874 .

11 La question de la qualité de la frappe monétaire est cruciale pour le Japon et l'ExtrêmeOrient. La volonté de l'empereur est d'éliminer les monnaies féodales, piètres, disparates et incohérentes. Pour s'imposer comme puissance économique la monnaie japonaise doit être au-dessus de tout soupçon. Les rapports publiés par Kinder puis par ses successeurs mettent tous en avant la qualité des espèces ${ }^{4}$. Il fait régulièrement prélever de façon aléatoire des monnaies dont il fait vérifier les poids et les titres à la fois à Londres et à San Francisco. Les rapports détaillent avec précision la faible tolérance dans les écarts des poids et des titres. L'objectif est de produire des monnaies de meilleure qualité que les ateliers occidentaux ou américains: c'est une condition sine qua non pour le développement du commerce japonais. Les négociants savent que le yen est alors la monnaie de référence, presque la monnaie officielle du commerce, dans tout l'Orient. Le transfert de technologie réussit : le 24 février 1880, cinq ans après le départ de Kinder, le Daily Press reproduit le débat à la chambre de commerce de Hong Kong:

Hon. P. Ryrie: there was a very long discussion on the Japanese yen in this Chamber. [...] This Chamber had not sufficient confidence in the Japanese Government as to their keeping up the purity of the coin. [...] I have myself had conversations on the subject with gentlemen who were well informed, and I have 
also had conversations on it with the late master of the Japanese Mint, Major Kinder, and I believe that the most perfect and reliable assurances can now be given by the Japanese Government that the purity of the coin will be kept up ${ }^{5}$. Meiji. Les guerres civiles qui opposent l'empereur aux shoguns récalcitrants dans les années 1870 sont alors financées par l'émission de papier monnaie, entraînant une inflation conséquente. La restauration des finances publiques devient l'œuvre de Matsukata Masayoshi, plusieurs fois ministre des finances et Premier ministre. L'une de ses tâches est de doter le Japon d'une banque nationale. Là encore c'est en Europe que Matsukata prend son modèle : les statuts de la Banque du Japon s'inspirent de ceux de la Banque de France. Il conduit en personne la délégation japonaise lors de l'Exposition universelle à Paris en 1878 et, s'il n'a pas été admis à la Conférence monétaire internationale d'août 1878, il se lie avec son organisateur Léon Say, Ministre des Finances du gouvernement français. Il met à profit son séjour pour étudier en détail la Banque de France et la Banque de Belgique auxquelles il fait allusion dans ses ouvrages ${ }^{6}$. En novembre 1883, quelques mois après la création de la Banque du Japon, il écrit à Léon Say :

As you may remember, it has been four years since I parted from you in 1879. I am still thankful for the kind consideration you gave me during my stay in your country. I particular benefited from you many useful suggestions on national finance [...]. Now as minister of finance, I am assuming the heavy responsibility of administering Japan's national finance and am truing my best, day and night, to put into effect what you kindly suggested?.

Passer des monnaies traditionnelles de cuivre coulé à des monnaies en argent était un premier choc. Cependant, dès 1870-1873, le cours de l'argent-métal s'effondre, perturbant tous les systèmes monétaires bimétalliques. Rapidement, les principaux pays optent, peu ou prou, pour un monométallisme or. L'Union Latine, cette entente monétaire bimétallique entre plusieurs pays européens créée en décembre 1865, est mise à mal et, dès juin 1867, les conférences internationales réclament l'adoption de l'étalon-or. Le refus des pays de l'Union Latine ouvre une période de crise monétaire : aucun État ne peut admettre de voir le prix de l'argent décrocher de celui de l'or. Les uns après les autres, les pays les plus riches sortent de l'Union pour adopter le monométallisme or. La crise de la dépréciation de l'argent touche aussi le Japon, peut-être un peu plus tardivement car le pays manquait cruellement d'argent et pouvait absorber de grandes quantités de métal.

apon des années 1890 n'est plus celui des années 1870 : il se comporte en puissance colonisatrice et guerrière, fort de ses progrès dans tous les domaines. À l'instar de l'Allemagne, le Japon ouvre les hostilités avec la Chine (1 ${ }^{\text {er }}$ août 1894-17 avril 1895) et exige de la Chine vaincue une indemnité payable en or à Londres (32900980 livres sterlings), environ 240 tonnes d'or monnayé. Comme l'Allemagne le fait en 1873 en adoptant pour le mark un étalon-or grâce à l'indemnité de guerre payée par la France, le Japon adopte le $1^{\text {er }}$ octobre 1897 le monométallisme or. Ainsi, le pays passe dans le camp des grandes puissances guerrières, dominatrices, à étalon-or.

L'enjeu d'une telle recherche n'est pas seulement de suivre la façon dont le transfert de compétence s'est effectué au bénéfice du Japon. L'intérêt de ces sources est en effet de fournir des éclairages croisés sur cette transformation. Le choc culturel que représente le changement de monnaie (passage à un système décimal, changement de la forme des espèces, changement des alliages, production industrielle avec des presses à vapeur, etc.), et donc de système de taxation, est documenté par des textes réglementaires, forts 
déshumanisés, et aussi par les nombreux ouvrages que le ministre Matsukata, le principal artisan des transformations, a laissés ${ }^{8}$. À ces sources s'ajoutent les rapports officiels, japonais, ou établis par les consulats et ambassades ou représentations commerciales des États étrangers à destination de leurs instances officielles9. Les journaux publiés au Japon pour les communautés d'expatriés fourmillent aussi d'informations : la presse étrangère ${ }^{10}$ , de diffusion réduite, était rédigée non pas par des journalistes professionnels mais par des personnes souvent impliquées dans des activités commerciales; auteurs et lecteurs étaient donc tous avides d'informations économiques. Enfin, les Japonais, dans leurs écrits, réagissaient parfois aux mesures économiques, fiscales et monétaires.

La documentation permet non seulement de comprendre l'exemplarité de la réforme (ou des réformes) économique, mais aussi de suivre les différentes perceptions, de différents points de vue, d'un transfert technologique. Nous sommes ainsi invités à effectuer un autre transfert culturel, si l'on peut dire, qui peut orienter la méthodologie de la recherche : à réfléchir à ce que la compréhension précise d'un phénomène particulier et documenté peut nous permettre de comprendre d'évolutions similaires lors de périodes moins documentées. Passer du koban au yen, du statère au denier, de la livre au franc, ce sont là diverses évolutions, diverses monnaies, diverses zones, diverses époque, etc. - mais tout cela est bien semblable. L'étude des transferts est souvent une opportunité de nous regarder avec les yeux des autres.

\section{ANNEXES}

Les photos reproduites ci-après ont été prises par Georges Depeyrot lors d'un passage à Osaka.

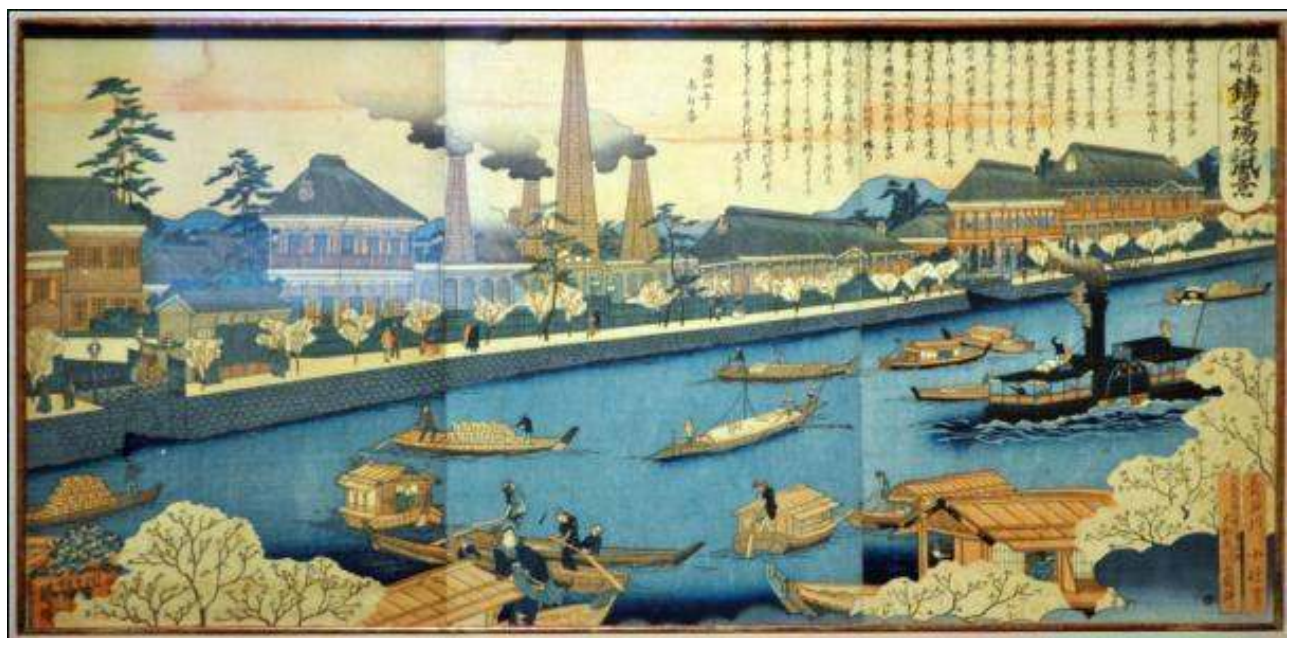

La monnaie d'Osaka, estampe, Musée de la Monnaie, Osaka. 


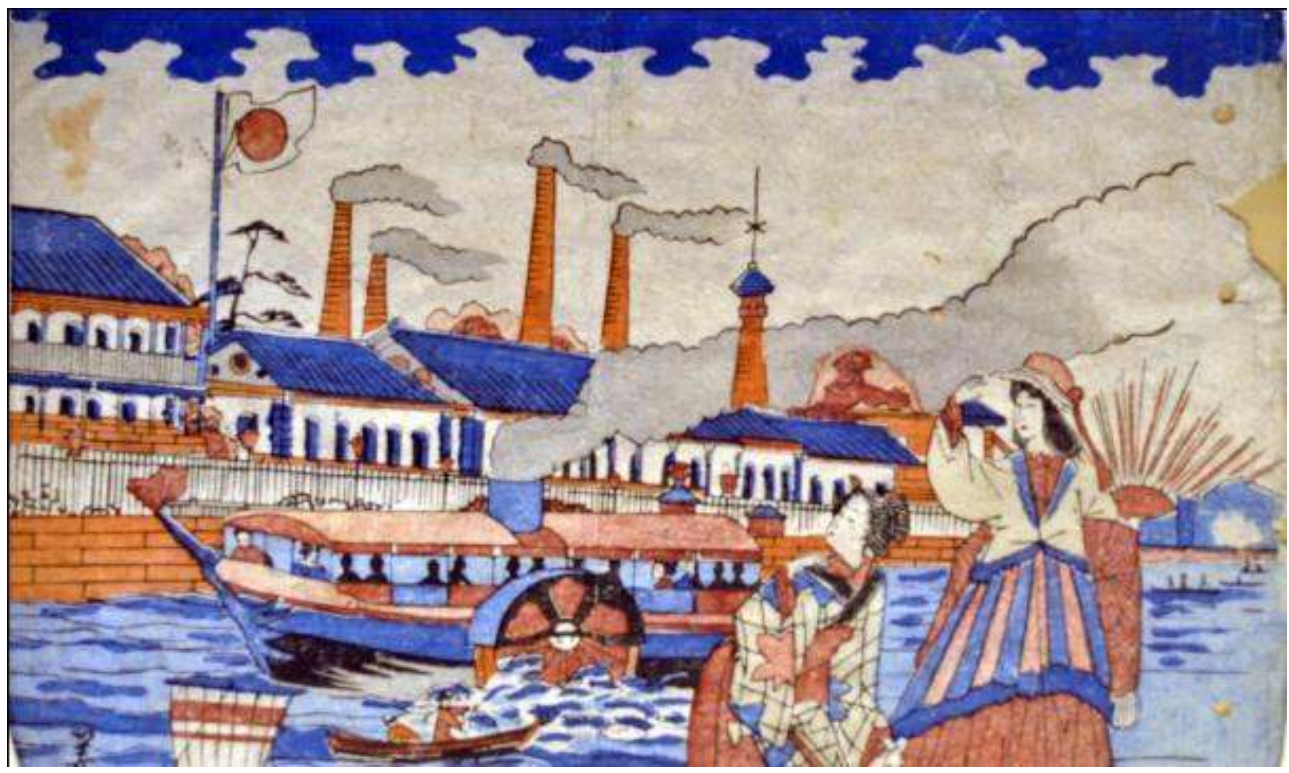

La monnaie d'Osaka, estampe, Musée de la Monnaie, Osaka.

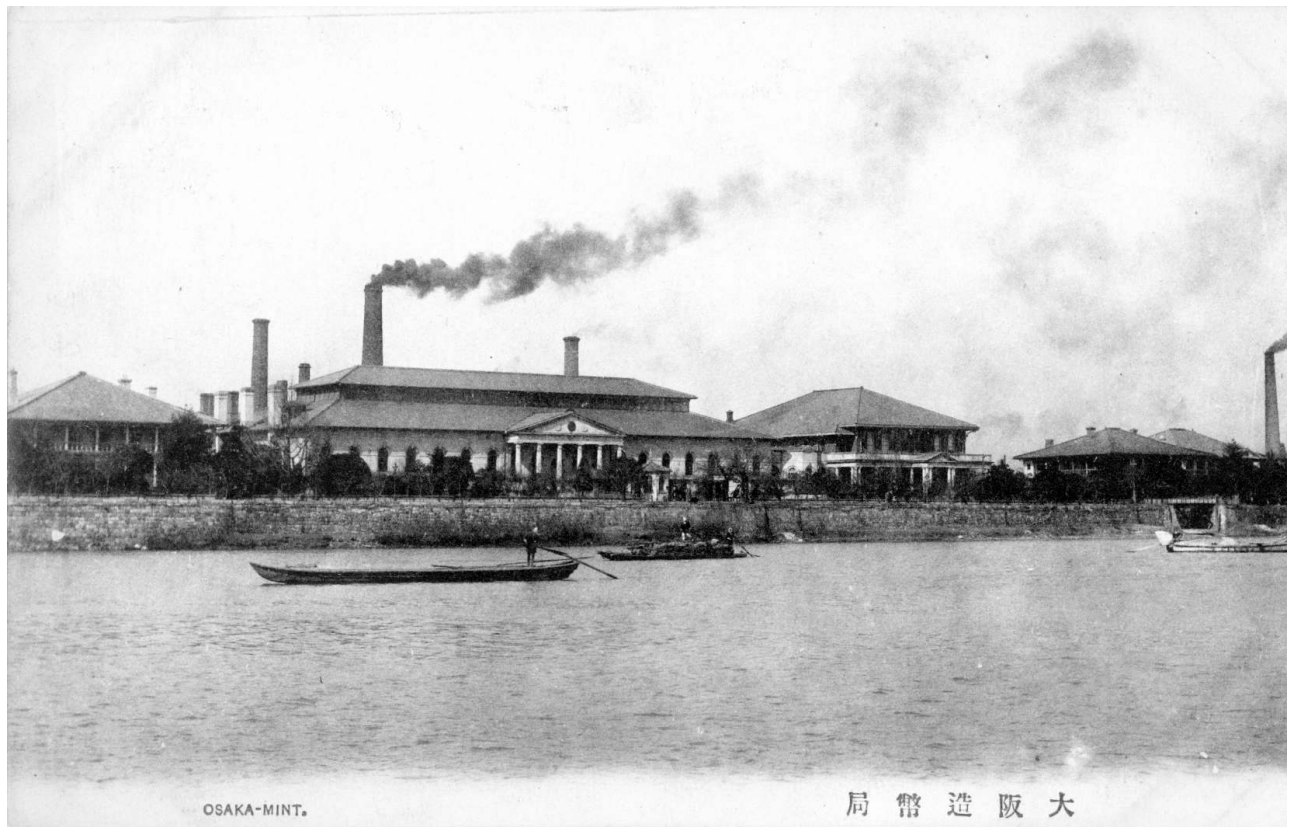

La monnaie d'Osaka, carte postale. 


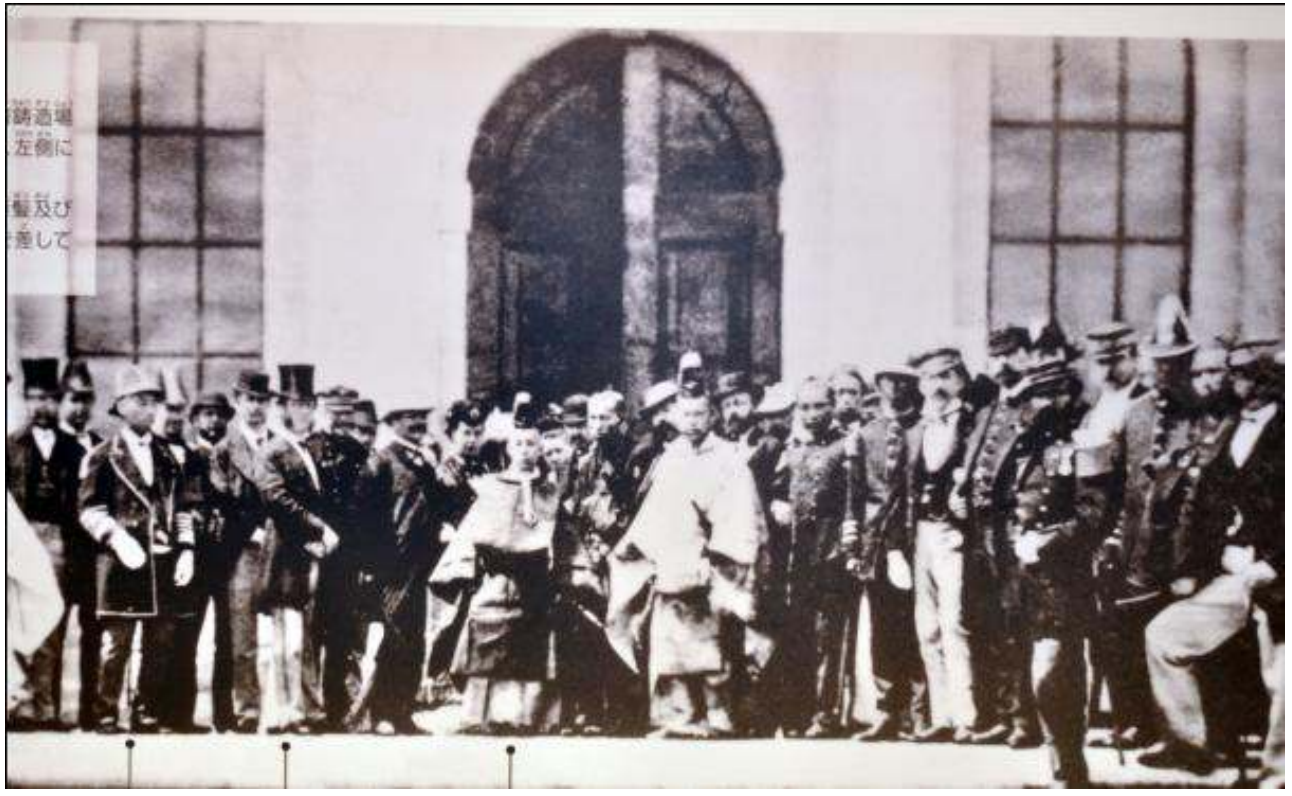

Inauguration de la Monnaie, le 4 avril 1871, Musée de la Monnaie, Osaka.

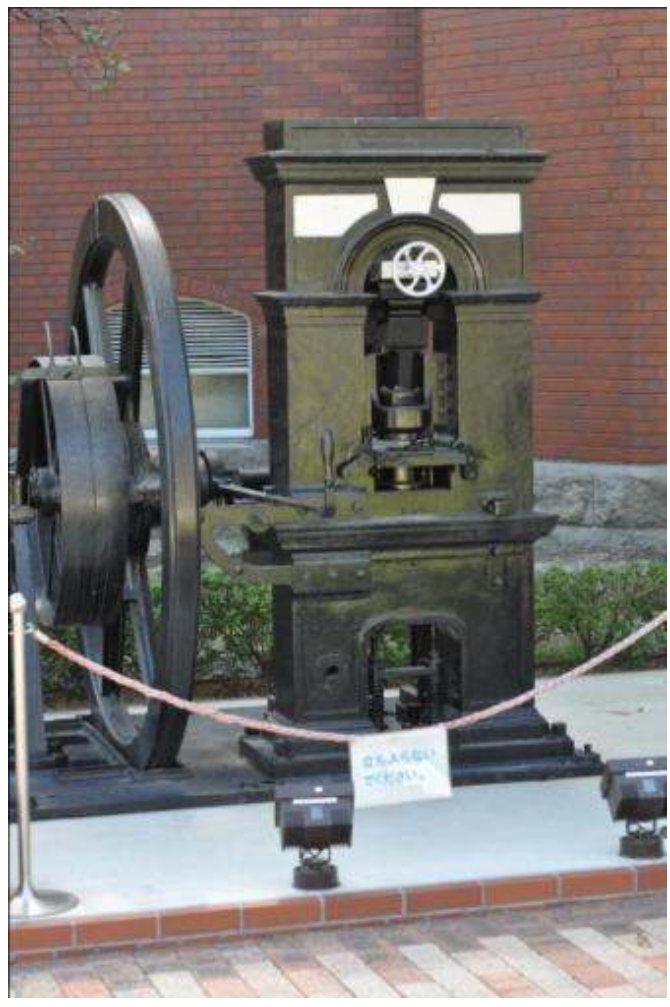

Presse française type Tonelli, 1871, Musée de la Monnaie, Osaka. 


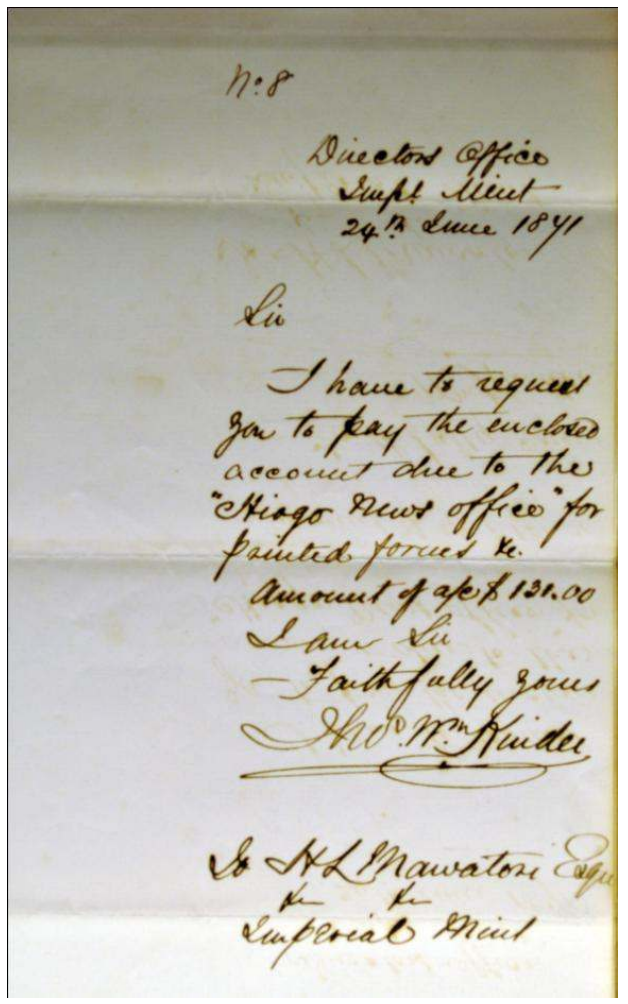

Lettre autographe de Thomas Kinder, 1871, Musée de la Monnaie, Osaka.

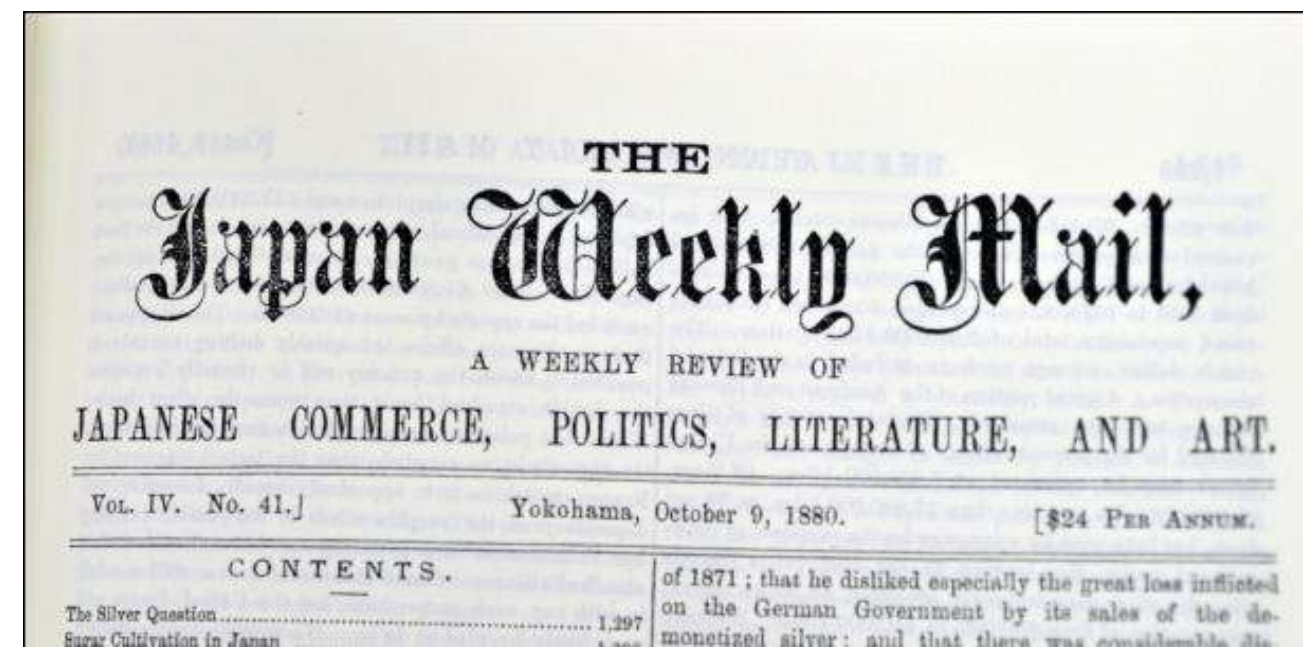

Japan Weekly Mail, un des journaux de la communauté anglophone à Yokohama, 1880, Bibliothèque d'Osaka (cet exemplaire titre sur la crise de l'argent).

\section{NOTES}

1. 特命全権大使米欧回覧実記 (1-5) 久米邦武 田中彰 (岩波文庫) 1977 ; traduits et publiés sous le titre Iwakura Embassy, 1871-1873. A True Account of the Ambassador Extraordinary and Plenipotentiary's Journey of Observation Through the United States of America and Europe, Graham Healey (dir.), Chushichi Tsuzuki, Londres, Routledge Curzon, 2002.

2. Document publié dans Count Matsukata Masayoshi, Report on the adoption of the Gold Standard in Japan, Tokio, Printed at the Government Press, Thirty Second Year of Meiji (1899), p. 5. 
3. Fourth Report of the Director of the Imperial Mint, Osaka, Japan, for the Half-Year ending $31^{\text {st }}$ January, 1875 , printed by order of the Council of State, Tokio, printed at the «Seishi-Bunsha » Office, 1878, p. 6.

4. Ces rapports s'intitulent Report of the Director of the Imperial Mint pour la période 1872-1875 lorsque Thomas Kinder était directeur ; puis Report of the Commissionner of the Imperial Mint, années 8-20 de l'ère Meiji (1875-1890), lorsque H. L. Mawatori, T. Ishimal et K. S. Endo étaient successivement commissionner of the Imperial Mint ; puis Report of the Director of the Imperial Mint , après 1891 , lorsque K. S. Endo fut nommé directeur.

5. Documents and Studies on $19^{\text {th }}$ c. Monetary History, Japan, Republication of The Currency of Japan, A Reprint of Articles, Letters, and official Reports, Published at Intervals in the Foreign Newspapers of Japan, together with Translations from Japanese Journals, Relating to the Currency, Paper and Metallic, of the Empire of Japan (Yokohama, 1882), Marina Kovalchuk, Georges Depeyrot (éd.), Wetteren, Collection Moneta, n 121, 2011, p 182.

6. Sur cette question, voir Michael Schiltz, «An ideal bank of issue : the Banque Nationale de Belgique as a model for the Bank of Japan », Financial History Review, 13 (2), 2006, p. 179-196, et 室 山義正, 松方正義一我に奇策あるに非ず、唯正直あるのみ, 2005.

7. Cette lettre de Matsukata a été traduite en anglais et publiée par Michael Schiltz, dans «An ideal bank of issue : the Banque Nationale de Belgique as a model for the Bank of Japan », article cité.

8. Les ouvrages de M. Matsukata étaient à la fois des justifications (à usage interne) de sa politique et des documents à destination des marchés financiers étrangers dont le Japon sollicitait les fonds. Voir :

Matsukata M. (松方正義), The History of National Debts in Japan (日本国債史) Tokyo, 1890.

Matsukata M., (松方正義), 紙幣整理始末 [Shihei Seiri Shimatsu]. Tokyo, 1890.

Matsukata M. (松方正義), Report on the Adoption of the Gold Standard in Japan (日本金本位制採用報 告), Tokyo, 1899.

Matsukata M. (松方正義), Report on the Post-bellum Financial Administration in Japan, (1896-1900) (戦 後財政始末報告), Tokyo, 1901.

9. C'est le cas des correspondances entre Londres et le Japon (Great Britain. Public Record Office. British Foreign Office, Japan. Correspondence, 1856-1905).

10. Plusieurs journaux ont été régulièrement publiés au Japon par et pour les expatriés, citons Japan Herald (créé en 1862), The Japan Times (créé en 1865), Japan Gazette (créé en 1867), Japan Weekly Mail (créé en 1870). Ils étaient principalement publiés à Yokohama. En 1882, le Japan Gazette publia un recueil de textes parus dans ses colonnes et dans les autres journaux sur la question financière. Il s'agissait d'inciter le gouvernement japonais à prendre des mesures drastiques contre l'inflation (voir Documents and Studies on $19^{\text {th }} \mathrm{c}$. Monetary History, Japan, éd. citée). Les réformes préconisées par le Japan Gazette furent appliquées par M. Matsukata, nommé ministre des finances en 1882 .

\section{RÉSUMÉS}

Lors de son ouverture au monde occidental (la « restauration Meiji »), le Japon s'est inspiré des modèles étrangers pour passer d'un système quasi-féodal à une organisation d'État moderne. Cette ouverture, préparée par des missions d'études en Europe ou aux États-Unis, a radicalement 
transformé le pays. Dans le domaine monétaire, la création d'un nouveau système et d'un stock monétaires, prélude aux changements fiscaux et bancaires, n'a été possible que par l'acquisition d'un atelier monétaire complet (celui de Hong Kong) et par un vaste transfert de savoir-faire technologique qui a permis au Japon de devenir une grande puissance financière.

When it opened to the Western world (during the "Meiji Restoration »), Japan drew its inspiration from foreign models in order to move from a quasi-feudal system to the organization of a modern state. This opening, prepared by study missions to Europe and the United States, completely transformed the country. In the monetary field, the creation of a new system and of a stock of money, paving the way for tax and banking changes, was only possible through the acquisition of a complete mint (that of Honk Kong) and a large transfer of technological knowhow, which enabled Japan to become a great monetary power.

\section{INDEX}

Mots-clés : monnaie, banque, Japon, transfert culturel

Keywords : currency, bank, Japan, cultural transfer

\section{AUTEURS}

\section{GEORGES DEPEYROT}

Chargé de recherches, CNRS, AOROC (UMR 8546, CNRS-ENS)

\section{MARINA KOVALCHUK}

Professeur associée, Far Eastern Federal University, Vladivostok

Parmi les publications :

Documents and Studies on $19^{\text {th }} \mathrm{c}$. Monetary History, Japan, Republication of The Currency of Japan, A Reprint of Articles, Letters, and Official Reports, Published at Intervals in the Foreign Newspapers of Japan, together with Translations from Japanese Journals, Relating to the Currency, Paper and Metallic, of the Empire of Japan (Yokohama, 1882), Marina Kovalchuk, Georges Depeyrot (éd.), Wetteren, C ollection Moneta, $\mathrm{n}^{\circ}$ 121, 2011. 